\title{
The effect of cue-fading on the DRL performance of septal and normal rats
}

\author{
PAUL ELLEN, PAMELA G. DORSETT, and W. KIRK RICHARDSON \\ Georgia State University, Atlanta, Georgia 30303
}

\begin{abstract}
The effect of a cue-light fading procedure on the subsequent noncued performance under a differential-reinforcement-of-low-rate schedule (DRL) of food reinforcement was determined for rats with septal lesions and normal rats. Following initial DRL-20 sec training, all animals were trained with a cued DRL schedule in which a light signaled the end of the required delay and the availability of reinforcement. Subsequently, for one-half of the animals, the cue light was abruptly discontinued; for the remainder of the subjects, the cue was gradually faded out over succeeding sessions. Septal animals, which had the cue gradually faded, were more efficient by the end of training than septal animals which had the light abruptly discontinued. Moreover, the former did not differ from normal controls. For septal animals in the Fade group, the relative frequency distributions of interresponse times (IRTs) were bimodal, a characteristic of normal animals, septal animals which had the cue abruptly terminated, displayed the unimodal distribution of IRTs which is a characteristic of septal animals on the DRL schedule. In summary, the cue-fading procedure completely eliminated the major differences in behavior between normal and septal rats typically observed on a DRL schedule of food reinforcement.
\end{abstract}

It is well documented that septal animals perform less efficiently, i.e., they emit more responses per reinforcement (response/reinforcement ratio) than do normals on a differential-reinforcement-oflow-rate (DRL) schedule (Burkett \& Bunnell, 1966; Ellen, Wilson, \& Powell, 1964). A DRL schedule requires that the animal not respond for some minimum period of time. At the end of the required interval, a response will deliver the reinforcer; if the response is made before the required time has elapsed, reinforcement is not delivered and the clock is recycled. However, high response/ reinforcement ratios are not an invariant consequence of septal damage. A variety of techniques have been shown to attenuate the inefficiency of septal animals on a DRL schedule. For example, cued DRL (Aitken, 1974; Braggio \& Ellen, 1976; Ellen \& Butter, 1969) with a light as the cue signaling the end of the required delay has been used to decrease the response/reinforcement ratios. Providing the animal with a wooden block and cardboard to chew (Slonaker \& Hothersall, 1972) has also produced more efficient septal performance on the DRL schedule. Braggio and Ellen (1974) further demonstrated that septal animals could perform similarly to normals when the force required to de-

This report is based upon a thesis submitted by the second author as partial fulfillment for requirements for the Master of Arts degree at Georgia State University, Atlanta, Georgia. Requests for reprints should be sent to Paul Ellen, Department of Psychology, Georgia State University, University Plaza, Atlanta, Georgia 30303. press the lever was increased or decreased from a particular value as a function of time since the last response.

Regardless of the technique used to reduce the response/reinforcement ratios of septal animals, the effectiveness of such procedures has been limited to the condition when the treatment was applied and did not carry over when the normal DRL procedure was reinstated. That is, performance reverted to the typical inefficient pattern when the additional stimuli were removed. Inasmuch as these response characteristics reappeared, Braggio and Ellen (1976) concluded that an inefficient performance on a DRL schedule was a primary consequence of the septal dysfunction and was not secondary to some other alteration resulting from the lesion.

It is the case, however, that those manipulations of the DRL situation which did facilitate efficient septal performance all involved increasing the stimulus control exerted by some stimulus dimension associated with the required response. Since under ordinary circumstances, the only differential stimulation available to the animal which could function to exert control over barpressing is that which arises from its own behavior, then perhaps, for the septal animal, inefficient performance on DRL reflects a failure of the response-produced stimulation to gain control over the behavior. Support for this view is found in the work of Caplan and Stamm (1967). These authors attempted to increase the control of response-produced stimuli over performance on the schedule by gradually shaping the animals into various DRL delays. Moreover, an efficiency cri- 
terion of $50 \%$ was required prior to the initiation of the next longer DRL requirement. Caplan and Stamm found that normal and septal animals which were gradually shaped into the longer delay requirements maintained comparable levels of efficiency throughout training, while septal rats which did not receive the shaping procedure performed less efficiently. It would thus appear that by gradually shaping the animals into the delay requirement, a sustained efficient performance for septal animals could be maintained even at DRL values which ordinarily produced high response/reinforcement ratios.

Other investigators have also used gradual shaping techniques during training to facilitate the development of stimulus control by a particular class of stimuli. One technique utilized, known as the easyto-hard procedure of stimulus fading, brings the organism to discriminate stimuli other than those which were discriminated initially. Evidence from studies of successive discrimination tasks with humans (Spiker, 1956) and simultaneous discrimination tasks with rats (Lawrence, 1952) suggests that a difficult discrimination, involving very similar stimuli, can be established by initially making the stimuli very different and then gradually making them more and more similar. Lawrence (1952) suggests that this procedure is effective because the relevant stimulus dimension, which is to acquire discriminative control over the behavior, is emphasized. As a result, stimulus control can be readily transferred across a range of changes in stimulus value. Thus, there is greater transfer of stimulus control when training is begun with distinctive stimuli. Retarded children have learned to discriminate different geometrical shapes without errors through the application of similar procedures (Sidman \& Stoddard, 1967).

The fading procedure has also been shown to be an effective technique for establishing stimulus control in animals with septal damage. Ellen and Powell (1966) implemented a fading procedure to develop differential stimulus control of two areas of brain stimulation. Originally, the animals were responsive to electrical stimulation of the septum, but were not responsive to hippocampal stimulation. Ellen and Powell paired stimulation of the two areas and gradually faded out septal stimulation as a discriminative stimulus such that hippocampal stimulation also acquired discriminative properties. Thus, through pairing and fading two distinct stimuli, discriminative control was transferred without a disruption in performance.

In short, the thrust of these latter studies strongly suggests that a gradual fading procedure may be beneficial in allowing stimuli, which would ordinarily fail to exert control over behavior, to become effective discriminative stimuli. Moreover, to the extent that such a fading procedure could sustain the improvement noted in animals with septal lesions when additional stimulation is provided, we would have obtained important evidence concerning the basis of the inefficient performance on the DRL schedule.

Therefore, the purpose of the present study was to determine whether or not the performance of animals with septal lesions on a DRL schedule could be maintained at the level of normals by gradually fading out a cue light which was used to signal the end of the required delay. The procedure involves a gradual transition from an easy to a more difficult discrimination, and a positive transfer from the cued to a noncued condition might be expected leading to sustained efficient DRL performance.

\section{METHOD}

\section{Subjects}

Sixteen naive Long-Evans hooded rats weighing 320-415 g prior to deprivation were used as subjects. Each animal was food-deprived to $85 \%$ of its free-feeding weight and maintained at that weight throughout the experiment.

\section{Surgery and Histology}

Eight rats were anesthetized with an IP injection of $40 \mathrm{mg} / \mathrm{kg}$ of sodium pentobarbital (Nembutal) and $.5 \mathrm{cc}$ of atropine sulfate. Each animal was placed in a Kopf stereotaxic device, and the scalp anesthetized with $2 \%$ lidocaine hydrochloride (Xylocaine) containing epinephrine. Stereotaxic coordinates were used (Pellegrino \& Cushman, 1967) with the electrode positioned $7.8 \mathrm{~mm}$ anterior to and $6.0-6.5 \mathrm{~mm}$ above the intraural line. Lesions were produced by passing $2 \mathrm{~mA}$ of anodal current from a Stoelting 58040 electrolytic lesion device for $20 \mathrm{sec}$ between the uninsulated tip of the electrode and a clip at the wound margin. Operated animals were then given $.2 \mathrm{cc}$ of procaine penicillin G (60,000 U, Duracillin) IM and allowed a 2- to 3-day recovery period before testing was begun.

After all testing was completed, the animals were sacrificed with chloroform and perfused intracardially with Ringer's solution followed by $10 \%$ Formalin. The brains were removed and fixed in Formalin for a 48-h period. They were then frozen and sliced $50 \mu \mathrm{m}$ thick, mounted on slides, and stained with cresyl violet.

\section{Apparatus and Procedure}

The animals were tested in Scientific Prototype operant chambers with retractable levers. The bars were located on the front wall of the chamber about $5 \mathrm{~cm}$ above the floor; they measured $5 \mathrm{~cm}$ in width $\times 1.3 \mathrm{~cm}$ thick and protruded $1.6 \mathrm{~cm}$ when fully extended into the chamber. A static force of $15 \mathrm{~g}$ through an excursion of $5 \mathrm{~mm}$ was required for a response to be recorded. Levers were inserted at the beginning of each session and retracted when the session terminated. A 45-mg Noyes food pellet was used as the reinforcer and was delivered to a food cup located approximately $2.5 \mathrm{~cm}$ to the left and $1.3 \mathrm{~cm}$ down from the lever. The only light in the chamber, a cue light (6-W $30-\mathrm{V}$ Westinghouse bulb behind a white translucent plastic cover), was located in the front of the chamber about $10.2 \mathrm{~cm}$ above the bar. The chambers were enclosed in wooden boxes with white noise in a soundproof room separate from the IBM 1800 computer system (Ellen, DeLoache, \& Bonds, 1972) that controlled the contingencies and recorded the data. 
The brightness of the cue light was controlled by varying the dc voltage to the bulb. Reductions in the voltage resulted in decreased brightness and yellowing of the light. Because the power supply used was not a constant voltage power supply, the cue light brightness varied slightly as a function of the number of chamber lights on. Voltage settings reported are those obtained with all of the lights on.

All subjects received the same initial training and were tested 7 days a week. The rats were reinforced for each response until a criterion of 150 barpresses for two consecutive 50-minute sessions was reached. Animals which did not spontaneously barpress during the first session were magazine trained and shaped to barpress. Subjects were given 20 sessions of DRL-20 training with no cue light, followed by 18 sessions of cued DRL-20. In the cued procedure, the light came on after $20 \mathrm{sec}$ elapsed without a response. The first barpress in the presence of the cue light was reinforced and the cue light was turned off. The manner of transition from cued to noncued DRL-20 differed for the two groups of normals and septals, i.e., the cue light was either gradually faded or abruptly discontinued. The Abrupt groups received a constant intensity cue, while the Fade groups were given gradually dimming cues over sessions. For the latter condition, initial voltage reductions were made according to the judgment of the experimenter that a change in brightness was observable but not expected to disrupt performance on the schedule. Thereafter, the decrements in voltage were much smaller. Conditions were changed when visual inspection of the response rates showed little or no systematic change. The conditions implemented and number of sessions for each condition are presented in Table 1.

Beginning with the first seven sessions of training at $2 \mathrm{~V}$ dc to the bulb, the subjects were allowed 10 min to dark-adapt in the chamber before testing was begun. For the remainder of the cue-faded conditions, 15 min of dark adaptation prior to testing was allowed. Finally, the cue was completely removed from both the Fade and the Abrupt groups.

\section{RESULTS}
Abrupt Group
Anatomical Findings
Lesions were generally large and located primarily in the midline, ventral septal area anterior to the anterior commissure. All animals received damage to the ventral medial septum and the diagonal band of Broca. There was some destruction of the medial and lateral preoptic areas and, to a lesser extent, of the hippocampal commissure and the anterior hypo- thalamic area. One animal suffered damage to the fornix.

\section{Fade Group}
Lesions were similar to those for the Abrupt group

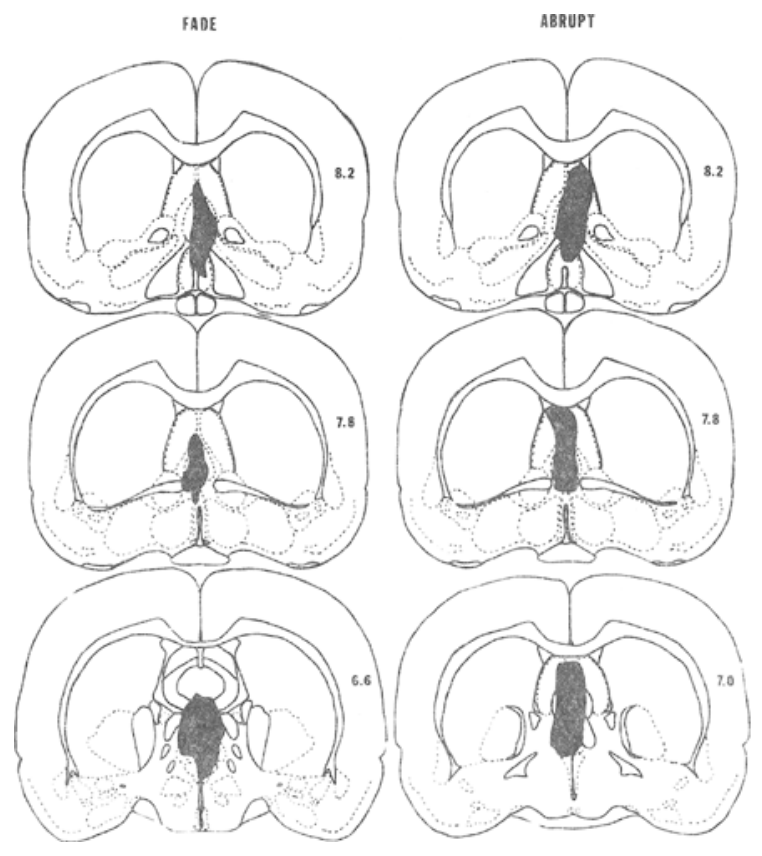

Figure 1. Reconstructions of sections to illustrate typical lesions for operated animals in fade and abrupt conditions. Sections include the area with primary damage to the septum as well as damage anterior and posterior to this area.

animals. Lateral and medial septum as well as medial and lateral preoptic areas and the diagonal band of Broca were damaged. There was destruction of the hippocampal commissure and anterior hypothalamic area. Two animals suffered damage to the anterior thalamic area. See Figure 1 for reconstruction of the lesions typical of each group.

\section{BEHAVIORAL FINDINGS}

\section{Responses per Reinforcement (Efficiency)}

Precue. Figure 2 represents the mean response/ reinforcement ratios for each of the four groups, Normal-Abrupt (NA), Normal-Fade (NF), SeptalAbrupt (SA), and Septal-Fade (SF), as a function of sessions for precue and postcue DRL-20 training. For Sessions 1-20, both SA and SF groups maintained higher efficiency ratios (91.9) than their normal counterparts (25.3). A two-way ANOVA (Days and Lesions) with repetition over Days (14-20)

Table 1

Training Conditions and Number of Sessions in Each Condition

\begin{tabular}{cccccc}
\hline & \multicolumn{5}{c}{ Training Conditions } \\
\cline { 2 - 6 } Group & DRL-20 & $\begin{array}{c}\text { Cued } \\
\text { DRL-20 }\end{array}$ & Cue Fade & $\begin{array}{c}\text { Cued } \\
\text { DRL-20 }\end{array}$ & DRL-20 \\
\hline Septal/Normal Fade & 20 & $18(26)$ & $\begin{array}{l}2(15), 2(12), 2(10), 2(8), 2(6), 2(4), \\
2(3), 12(2), 2(1.5), 2(1.0), 4(.5)\end{array}$ & 12 \\
Septal/Normal Abrupt & 20 & $18(26)$ & & $34(26)$ & 12 \\
\hline
\end{tabular}

Note-Numbers in parentheses indicate dc volts used to illuminate cue light. During initial training, all animals received a continuous reinforcement schedule to a criterion of 150 barpresses for two consecutive 50-min sessions. 


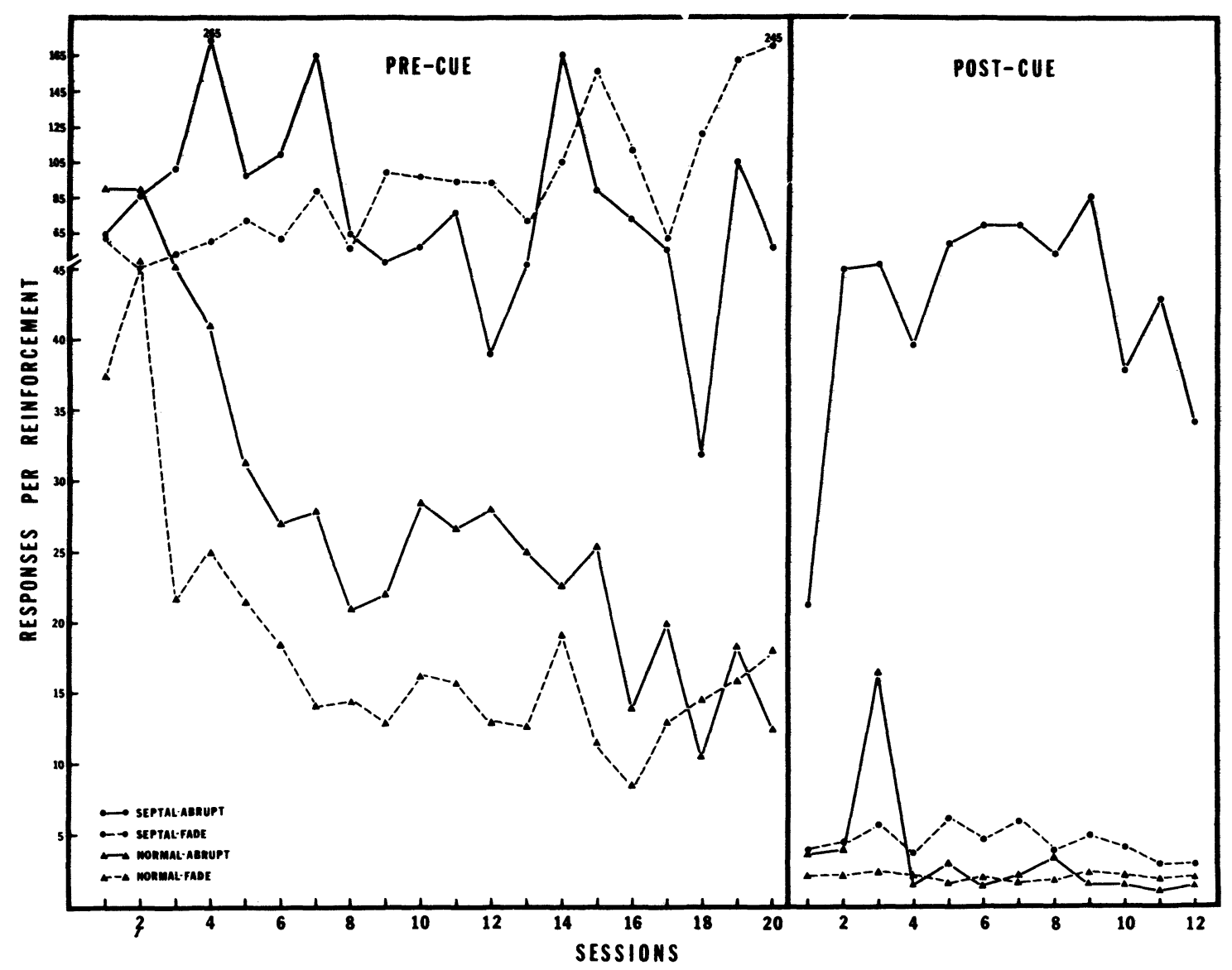

Figure 2. Mean number of responses per reinforcement during DRL for each of the four groups prior to initial cue training and after the cue procedure was discontinued.

indicated that the septal-normal difference was significant, $[F(1,14)=5.02, p<.05]$. There was no significant Days effect, indicating that the behavior had stabilized. Furthermore, no significant differences were found within groups by this point in training, as indicated by a two-way ANOVA (Conditions and Days) with repetition over Days (14-20).

Cue. With introduction of the cue light, response/ reinforcement ratios decreased for all groups and the septal animals did not differ from the normal controls. By the last four sessions of this phase of the experiment and prior to the onset of the fading procedure, normal animals in the two conditions emitted $1.21 \pm .15$ (abrupt) and 1.44 \pm .24 (fade) responses per reinforcement. Septal animals in the abrupt condition made $1.39 \pm .40$ responses per reinforcement during the cued-DRL condition and rats in the fade condition generated a mean efficiency ratio of $1.52 \pm .51$. A three-way ANOVA (Days, Condition, and Lesions) with repetitions over the last 4 days of the cued condition (17-20) indicated that the septal-normal difference was not significant $[F(1,12)=.52, p>.05]$ and that fade-abrupt condition differences were not significant $[\mathrm{F}(1,12)$ $=1.13, \mathrm{p}>.05]$. More importantly, there were no significant interactions. It is clear from these data that for all groups the cue condition reduced the $\mathrm{R} / \mathrm{Rf}$ ratios and that there were no differential effects of the cueing due to the presence of the lesion.

It will be recalled that after 18 days of cueing with the cue light voltage set at $26 \mathrm{~V} \mathrm{dc}$, one septal and one normal group were exposed to the fading procedure while the voltage to the light was gradually reduced. The remaining two groups continued to receive a cue light of $26 \mathrm{~V}$ dc. During the fading procedure, mean efficiency ratios remained relatively stable throughout all light intensities for both faded groups. As indicated in Table 2 , the mean $\mathbf{R} / \mathbf{R f}$ ratios for each dc voltage value for normal and septal rats in the fade condition were comparable with the values obtained by the animals in the abrupt condition during these sessions. It is of interest to note that even when the voltage to the cue light was reduced to $0.5 \mathrm{~V} \mathrm{dc}$, there was still no marked decrease in efficiency as occurred when the cue was removed abruptly (see below) for the remaining two groups. 
Table 2

Mean Response/Reinforcement Ratios Across Fading Sessions

\begin{tabular}{cccccccccccc}
\hline & \multicolumn{10}{c}{ dc Voltage Reductions } \\
\cline { 2 - 12 } Group & 15 & 12 & 10 & 8 & 6 & 4 & 3 & 2 & 1.5 & 1.0 & .5 \\
\hline Septal & & & & & & & & & & & \\
Fade & 1.5 & 1.4 & 1.4 & 1.1 & 1.4 & 1.5 & 1.5 & 1.6 & 1.5 & 2.1 & 2.6 \\
Abrupt & 1.4 & 1.4 & 1.1 & 1.2 & 1.2 & 1.3 & 1.2 & 1.5 & 1.9 & 2.1 & 1.8 \\
Normal & & & & & & & & & & & \\
Fade & 1.4 & 1.3 & 1.3 & 1.4 & 1.3 & 1.4 & 1.6 & 1.6 & 1.5 & 1.6 & 1.9 \\
Abrupt & 1.2 & 1.1 & 1.1 & 1.0 & 1.2 & 1.1 & 1.1 & 1.1 & 1.1 & 1.1 & 1.1 \\
\hline
\end{tabular}

Note-Animals in the abrupt groups continued to receive a $26-V$ dc cue light during this phase.

Postcue. Upon cue termination, responses per reinforcement increased dramatically for the SA group but only slightly for the remaining groups (Figure 2, right-hand panel). Furthermore, the SA response/reinforcement ratio continued to increase over days while the NA group rapidly recovered a low response/reinforcement ratio. Both Fade groups (septals as well as normals) displayed little effect of cue removal and remained stable throughout the terminal DRL training. A three-way ANOVA (Days, Condition, and Lesion) with repetition over the Days factor (Days 9-12) supported these impressions. There was a significant Days effect $[F(3,36)=4.41, p<.01]$, Days by Condition interaction $(F=3.73, p<.05)$, Days by Lesions interaction $(F=4.29, p<.01)$, and triple interaction $(F=3.80, p<.05)$, thus indicating that there was a change over days and that this change was differential depending upon the condition and the lesion. Tests of all possible planned comparisons of lesioncondition cell means for Sessions 9-12 further substantiated the SA group differences. Efficiencies for the SA animals significantly differed from NA $[F(1,12)=5.37, p<.05], N F(F=5.25, p<.05)$, and $\mathrm{SF}(\mathrm{F}=4.90, \mathrm{p}<.05)$ performances. Hence, although the SA and SF groups initially performed at similar levels of efficiency, by the end of DRL training the two groups were significantly different. Furthermore, SF group response/reinforcement ratios were not significantly different from either of the two normal groups by this point in training.

\section{Relative Frequency Distribution}

Figure 3 represents the mean IRT distributions in 2-sec class intervals for all groups over the last four sessions of precue training (3A), cued training (3B), and postcue training (3C). The data in Figure 3B do not include that generated during the fading procedure. For initial DRL training (3A), the relative frequency distributions of septals are unimodal, with the greatest percentage of responses occurring at the shortest IRT interval and decreasing as the IRT increases. In contrast, both of the distributions for the normal animals are bimodal with the second mode at or around the required delay.
Figure 3B indicates that all groups exhibited bimodal distributions with greatest responding at the end of the required delay and an initial mode at the first or second IRT interval for the last four sessions of the cue procedure prior to the onset of fading.

Upon cue termination, the bimodal form of the relative frequency distributions were generally maintained for all but the SA group, which showed a dramatic increase in the frequency of IRTs below the required DRL value. For the last four sessions of training (Figure 3C), the SA group displayed the unimodal distribution with no peak at the DRL value, similar to the distribution of $3 \mathrm{~A}$. Relative to the initial DRL training, the SF group distribution shifted such that by the end of training it resembled that of normal animals. Hence, the fading procedure served not only to increase the frequency of long IRTs during cue training, but the performance was sustained after termination of the cue.

\section{IRTs per Opportunity Statistic}

The right panels of Figure 3 (D-F) present the conditional response probabilities (IRTs/Op) for each of the four groups averaged over the last four sessions of precue training (3D), cue training (3E), and postcue training (3F). The IRTs/Op statistic (Anger, 1956) takes into consideration the opportunity of an IRT of a particular delay relative to the occurrence of other IRTs.

Prior to cue training (Figure 3D), septal groups showed a flatter IRTs/Op function than normals. During cue training, all groups exhibited the greatest response probability at the reinforced IRT (see Figure 3E). For the last sessions of postcue training (Figure 3F), all groups display much steeper IRTs/Op functions than the SA animals, which retain a high response probability of nonreinforced IRTs. Thus, the conditional response probability of the SF group shifted over training so as to resemble the normal distribution, while the IRTs/Op function of the septal animals in the abrupt condition reverted to a flatter function similar to that observed after the initial noncued training. 


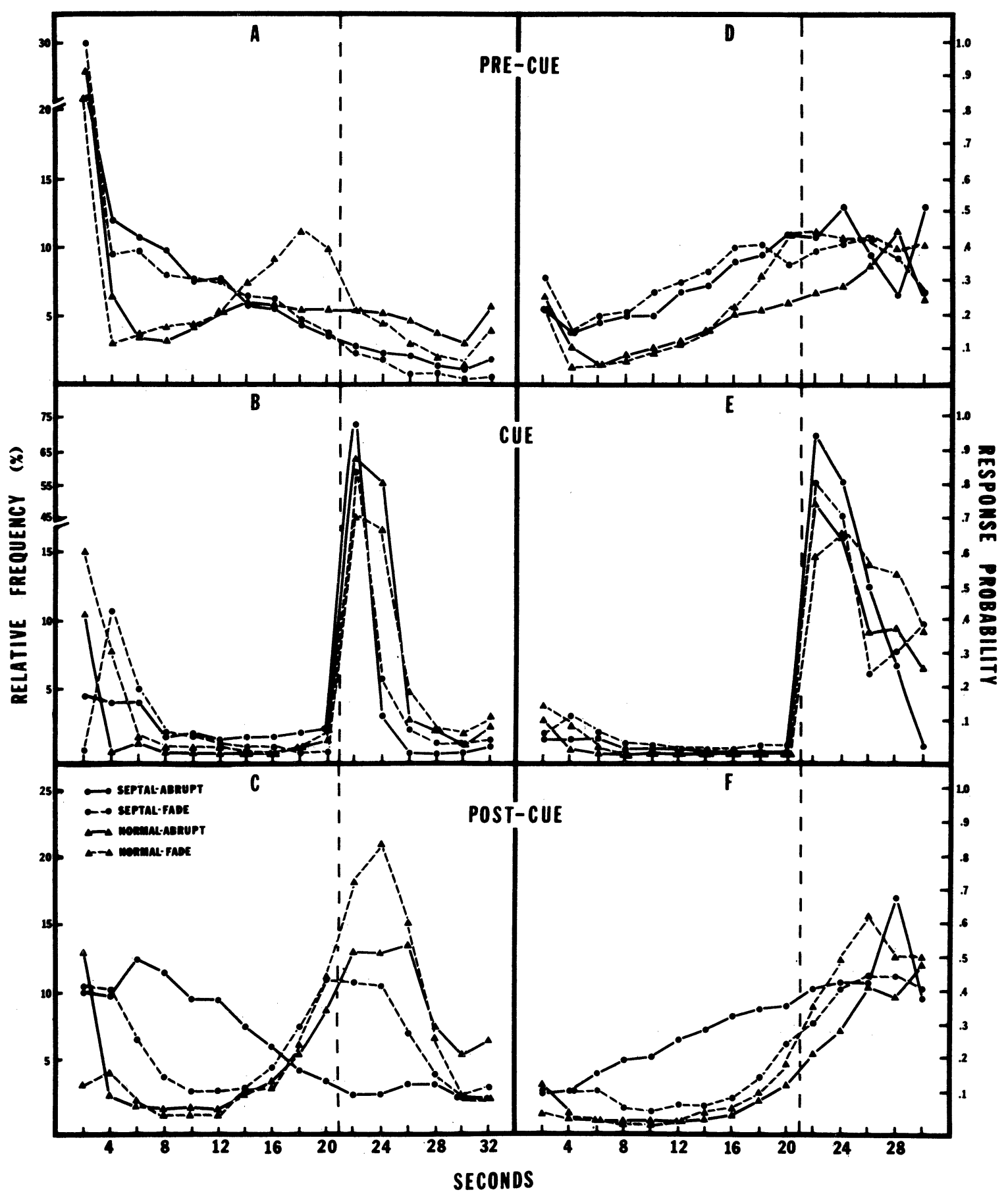

Figure 3. Mean relative frequency (Panels A-C) and mean response probability (Panels D-F) for each of four groups during DRL-20 prior to cue training (top row), during cue training (middle row), and subsequent to cue termination (bottom row).

\section{DISCUSSION}

The behavioral characteristics observed in the operated animals during the initial DRL training and following abrupt cue termination were indistinguishable from those shown by animals which sustain typical medial septal lesions (Braggio \& Ellen, 1976). The lesions in the present study extended ventrally from the medial septum into the diagonal band of Broca and lateral and medial preoptic areas, and thus the behavioral data obtained are consistent with the Ross and Grossman (1975) hypothesis, 
which suggests that the typical overresponding characteristics on a DRL schedule of animals with septal damage are due to disruption of septohippocampal connections traversing the medial septum and originating in the area of the diagonal band (Raisman, 1966).

However, the major finding of this study is the fact that following the cue fading procedure, septal animals did not differ from normals on any measure of DRL performance. The efficiency ratios, relative frequency, and IRTs/Op distributions of septals in the Fade group were indistinguishable from those generated by normals. Performance of these animals indicated that there was a positive transfer of stimulus control from the cued to the noncued condition and that this transfer could not be made by the septal animals without a gradual fading procedure.

The fact that the inefficiency of septal animals on DRL could indeed be eliminated provides evidence that this behavioral characteristic is not an invariant consequence of septal damage as originally suggested by Braggio and Ellen (1976). There are two important implications of this observation. First, since response inefficiency does not invariantly follow septal damage, it must not be a primary symptom of the damage. Rather, it would appear that inefficient responding on the DRL schedule is secondary to some other dysfunction and requires particular testing or training conditions for its appearance. Thus, response inefficiency would be a poor candidate for making assumptions about the role of the septum in behavior, since its appearance depends on details of the behavioral procedures used.

Secondly, our work indicates that the method of training septal animals on the DRL schedule may be a major determining factor in the acquisition and maintenance of efficient performance. Previous attempts to alleviate the septal deficit on DRL have involved abrupt introduction and/or termination of some particular stimulus conditions and consequently have not resulted in a sustained improved performance once such procedures were discontinued (Braggio \& Ellen, 1974, 1976; Ellen \& Butter, 1969; Slonaker \& Hothersall, 1972). The present results, in concert with Caplan and Stamm (1967), would suggest that if the treatment procedure involves an easy-to-hard discrimination technique, efficient DRL performance can be maintained after the training procedure has been discontinued. That is, by gradually increasing the DRL delay (shaping) or by gradually decreasing the value of an external stimulus which sets the occasion for responding along some continuum (fading), stimulus control is transferred and a sustained improvement in performance is obtained after the removal of the cue.

The results of this study do not clarify how or why a gradual elimination of stimuli is important for efficient DRL performance. However, those procedures which have been effective in producing longlasting treatment effects are characterized primarily by stimulus control factors. To the extent that such procedures facilitate the maintenance of treatment effects subsequent to termination, the importance of stimulus control in DRL performance is strongly suggested. It may be the case that these procedures are effective because they provide a mechanism for enhancing the discriminative properties of those stimuli necessary for adequate DRL performance.

But what are the stimuli which must gain control over the behavior? It will be recalled that under the DRL schedule there are no explicitly programmed external stimuli to set the occasion for barpressing (Ellen \& Butter, 1969). As a result, response-produced feedback would appear to be the only stimulation available to the animal to control its behavior. Judging from the relatively low level of reinforcements obtained by normal $(28 \%)$ as well as septal animals $(16 \%)$ in the present study, such feedback is, at best, a poor mechanism for establishing stimulus control over DRL responding. However, there are a number of lines of evidence which suggest that the attending of septal animals is restricted to the more salient and/or constant stimuli operating in a test situation (Dalland, 1974; Thomas, 1972). Thus, to the extent that the animal is responding on the basis of whatever exteroceptive stimuli are present in the chamber, the less salient response-produced stimulation would fail to gain control over the behavior. However, by pairing a highly specific and distinctive external stimulus associated with reinforcement and then gradually removing it, the control exerted by the exteroceptive stimulus can be effectively transferred to the response-produced stimuli without causing a disruption in efficient performance. This view would lend support to a hypothesis of septal dysfunction which proposes an attentional type of deficit.

Moreover, there is an interesting corollary to this approach. To the extent that it is possible to devise a treatment which not only leads to an improvement in behavior while it is being applied, but also allows a sustained improvement to be maintained when the treatment is removed, the possibility is afforded of determining those aspects of the lesion-induced disorder which indeed reflect a functional role of the damaged structure rather than the peculiarities of the testing situation.

Finally, it should be mentioned that no attempt is made here to allege that the cue-fading procedure has affected other aspects of the septal dysfunction (Fried, 1972). Rather, we are simply demonstrating that one aspect of the dysfunction, i.e., high response/ reinforcement ratios on operant schedules such as DRL, can be totally eliminated under appropriate 
training conditions. It remains to be determined whether animals which have shown such recovery following the cue-fading training would be impaired in other operant situations.

\section{REFERENCES}

AITKEN. W. C. The role of cues and feedback in the DRL performance of rats with septal lesions. Unpublished doctoral dissertation, Georgia State University, 1974.

ANGER, D. Dependence of interresponse times upon relative reinforcement of different interresponse times. Journal of Experimental Psychology, 1956, 52, 145-161.

Braggio, J. T., \& Ellen, P. Differential proprioceptive feedback and DRL performance of normal and septal rats. Journal of Comparative and Physiological Psychology, 1974, 87, 80-89.

Braggio, J. T., \& Ellen, P. Cued DRL training: Effects on the permanence of lesion-induced overresponding. Journal of Comparative and Physiological Psychology, 1976, 90, 694-703.

Burkett, E. E., \& BunNel, B. N. Septal lesions and the retention of DRL performance in the rat. Journal of Comparative and Physiological Psychology, 1966, 62, 468-471.

Caplan, M., \& STAMm, J. DRL acquisition in rats with septal lesions. Psychonomic Science, 1967, 8, 5-6.

Dalland, T. Stimulus perseveration of rats with septal lesions. Physiology and Behavior, 1974, 12, 1057-1061.

ELLEN, P.,\& BUTTER, J. External cue control of DRL performance in rats with septal lesions. Physiology and Behavior, 1969, 4, 1-6.

Ellen, P., DELoAChe, H., \& Bonds, J. Time-shared control of a variety of psychological laboratories using IBM 1800 data acquisition and control computer. Behavior Research Methods \& Instrumentation, 1972, 4, 81-85.
Ellen, P., \& Powell, E. W. Differential conditioning of septum and hippocampus. Experimental Neurology, 1966, 16, 162-171.

Ellen, P., Wilson, A. S., \& Powell, E. W. Septal inhibition and timing behavior in the rat. Experimental Neurology, 1964, 10, 120-132.

Fried, P. A. The septum and behavior: A review. Psychological Bulletin, 1972, 78, 292-310.

LAWRENCE, D. H. The transfer of a discrimination along a continuum. Journal of Comparative and Physiological Psychology, 1952, 45, 511-516.

Pellegrino, L. J., \& Cushman, A. J.A stereotaxic atlas of the rat brain. New York: Meredith, 1967.

Raisman, G. The connections of the septum. Brain, 1966, 89, 317-348.

Ross, J. F., \& Grossman, S. P. Septal influences on operant responding in the rat. Journal of Comparative and Physiological Psychology, 1975, 89, 523-535.

Sidman, M., \& Stoddard, L. L. The effectiveness of fading in programming a simultaneous form discrimination for retarded children. Journal of the Experimental Analysis of Behavior, 1967, 10, 3-15.

Slonaker, R., \& Hothersall, D. Collateral behaviors and the DRL deficit of rats with septal lesions. Journal of Comparative and Physiological Psychology, 1972, 80, 91-96.

SPIKER, C. C. Effects of stimulus similarity on discrimination learning. Journal of Experimental Psychology, 1956, 51, 393-395.

Thomas, J. B. Stimulus perseveration and choice behavior in rats with septal lesions. Journal of Comparative and Physiological Psychology, 1972, 80, 97-105.

(Received for publication April 18, 1977; revision accepted October $4,1977$. 